\title{
Prognostic values of EORTC QLQ-C30 and QLQ-HCC18 index-scores in patients with hepatocellular carcinoma - clinical application of health-related quality-of-life data
}

Leung Li ${ }^{1}$, Frankie KF Mo ${ }^{1}$, Stephen L Chan ${ }^{1}$, Edwin P Hui ${ }^{1}$, Nelson SL Tang ${ }^{4}$, Jane Koh ${ }^{1}$, Linda KS Leung ${ }^{1}$, Annette NY Poon ${ }^{1}$, Joyce Hui ${ }^{2}$, Cheuk M Chu ${ }^{2}$, Kit F Lee ${ }^{3}$, Brigette BY Ma', Paul BS Lai ${ }^{3}$, Anthony TC Chan?', Simon $\mathrm{CH} \mathrm{Yu}^{2}$ and Winnie $\mathrm{Yeo}^{1 *}$

\begin{abstract}
Background: Health-related quality-of-life (HRQOL) assessment with EORTC QLQ-C30 was prognostic for overall survival (OS) in patients with advance-stage hepatocellular carcinoma (HCC), but no data existed for early-stage patients. The HCC-specific QLQ-HCC18 has not been evaluated for prognostic value in HCC patients. Utilization of raw HRQOL data in clinical setting has been impractical and non-meaningful. Therefore we developed index scores of QLQ-C30 and QLQ-HCC18 in an attempt to enable clinical utilization of these HRQOL measurements. This study investigates the prognostic significance of QLQ-C30, QLQ-HCC18 and C30/HCC18 index-scores in patients with newly diagnosed HCC which encompasses all stages.

Methods: From 2007-2011, 517 patients were prospectively recruited. HRQOL was assessed at diagnosis using QLQ-C30 and QLQ-HCC18; C30 and HCC18 index-scores were calculated from raw HRQOL data. Cox regression was performed using continuous, dichotomized QLQ-C30 and QLQ-HCC18 variables, or index-scores, together with clinical factors to identify independent factors for OS. Various multivariate models were validated with c-index and bootstrapping for 1000 replications.
\end{abstract}

Results: Four hundred and seventy two patients had complete HRQOL data. Their median OS was 8.6 months. In multivariate analysis, independent prognostic HRQOL variables for OS were QLQ-C30 pain (HR 1.346 [1.092-1.661], $p=0.0055)$, QLQ-C30 physical functioning (HR 0.652 [0.495-0.860], $p=0.0024)$ ) QLQ-HCC18 pain (HR 1.382 [1.089-1. 754], $p=0.0077$ ) and QLQ-HCC18 fatigue (HR 1.441 [1.132-1.833], $p=0.0030)$. C30 index-score (HR 2.143 [1.616-2. 841], $p<0.0001)$ and HCC18 index-score (HR 1.957 [1.411-2.715], $p<0.0001)$ were highly significant factors for OS. The median OS of patients with C30 index-score of $0-20,21-40,41-60,61-100$ were $16.4,7.3,3.1,1.8$ months respectively $(p<0.0001)$; while for HCC18 index-score: $16.4,6.0,2.8,1.8$ months respectively $(p<0.0001)$. All the multivariate models were validated, with mean optimism $<0.01$. The bootstrap validated $c$-index was 0.78 .

\footnotetext{
* Correspondence: winnie@clo.cuhk.edu.hk

${ }^{1}$ Comprehensive Cancer Trials Unit, Department of Clinical Oncology, State Key Laboratory in Oncology in South China, Prince of Wales Hospital, Faculty of Medicine, The Chinese University of Hong Kong, Shatin, Hong Kong SAR Full list of author information is available at the end of the article
} 
(Continued from previous page)

Conclusions: QLQ-C30 and QLQ-HCC18 were prognostic for OS in patients with newly diagnosed HCC irrespective of stage. Both C30 and HCC18 index-scores were highly significant prognostic factors for OS in newly diagnosed HCC patients. Index-scoring provides an effective way to summarize, analyze and interpret raw HRQOL data, and renders QLQ-C30 and QLQ-HCC18 meaningful and communicable in clinical practice. Index-scores could potentially serve as a standardized tool for future HRQOL research.

Keywords: Health-related quality-of-life, QLQ-HCC18, QLQ-C30, Index-score, Prognosis, Overall survival, Hepatocellular carcinoma, Liver cancer

\section{Background}

Three studies have shown health-related quality-of-life (HRQOL) being prognostic for overall survival (OS) in patients with advance-stage hepatocellular carcinoma (HCC) [1-3]. These used general cancer HRQOL measurement tools, namely the European Organization for Research and Treatment of Cancer (EORTC) QLQ-C30 [4] and Spitzer QOL index [5]. On the other hand, one negative study recruited both early- and advance-stage HCC patients and used another general cancer HRQOL measurement, Functional Assessment of Cancer Therapy - General (FACT-G) [6, 7]. To date, there has been no study evaluating the prognostic value of QLQ-C30 for patients with newly diagnosed HCC which encompasses all stages.

Patients with HCC often suffer from chronic liver disease. In Asia, this is mainly due to chronic hepatitis B virus (HBV) infection [8-10]. Therefore liver-specific HRQOL measurement could be more relevant for these patients. EORTC QLQ-HCC18 [11] is a specific HRQOL module which addresses QOL issues specific for patients with primary liver cancer. It has been validated in Asian HCC patients $[12,13]$ and many scales of QLQ-HCC18 have been reported to enable the identification of patients with different clinical conditions. However, the prognostic value of EORTC QLQ-HCC18 in HCC patients has not been evaluated.

So far it has been a common practice to analyze raw HRQOL data as a collection of continuous variables, and various HRQOL factors have been proven to be prognostic for survival in various malignancies. Despite the wide utilization of EORTC QLQ-C30, there has been no domain/item identified to be consistently prognostic [14]. Difficulties in HRQOL research were well recognized: multi-collinearity among numerous raw HRQOL data causing multivariate analysis model instability, overfitting of variables leading to excessive multiple comparisons and type I error [14, 15], and lack of means to meaningfully translate raw HRQOL data into clinical use. Diouf et al. dichotomized all HRQOL data at a universal cut-off at 50 for analysis. This addressed the issues of multi-collinearity and overfitting and provided a way to interpret HRQOL data by clinicians [3]. A separate analysis was performed to determine the true cut-off for various domains/items, and these cut-offs have been considered to be potentially population-specific [16].

In an attempt to determine a generalizable way to analyze and interpret HRQOL data while minimizing multi-collinearity and over-fitting, we derived two index-scores, namely the C30 and HCC18 index-scores, to represent all domains and items within the EORTC QLQ-C30 and QLQ-HCC18 respectively.

The objectives of this study are: (1) to evaluate the prognostic value of QLQ-C30 in a prospective cohort of newly diagnosed patient with $\mathrm{HCC}$ which encompasses all stages; (2) to investigate the prognostic significance of the liver-specific QLQ-HCC18 in this cohort; and (3) to evaluate the prognostic significance of $\mathrm{C} 30$ and $\mathrm{HCC} 18$ index-scores.

\section{Methods}

From January 2007 to December 2011, all patients with newly diagnosed HCC presented to the multidisciplinary hepatoma clinic of Prince of Wales Hospital were considered for recruitment. The study was approved by the Joint Chinese University of Hong Kong-New Territories East Cluster Clinical Research Ethics Committee.

Eligibility criteria included: adult patients with newly diagnosed and treatment-naïve HCC; the diagnosis of HCC as confirmed by either histology, the combination of radiological and biochemical findings (space-occupying lesion in the liver with raised $\alpha$-fetoprotein (AFP $\geq 400 \mathrm{ug} /$ L), or 2 typical radiological findings with ultrasonography, triphasic computed tomography, angiography or magnetic resonance imaging; ability to read and comprehend Chinese was a pre-requisite. Patients were excluded if they had history of malignancy, encephalopathy or cognitive impairment.

\section{Treatment}

After confirmation of diagnosis and stage, patients were offered appropriate treatment as clinically indicated. Treatment options included surgical resection, local ablative therapies - radiofrequency ablation (RFA) or percutaneous ethanol injection (PEI), transarterial therapies transarterial chemo-embolisation (TACE) or transarterial 
injection of lipiodol-ethanol mixture (LEM), systemic therapies - sorafenib, chemotherapy, clinical trials and best supportive care (BSC) alone.

\section{HRQOL assessment}

Consented patients would complete two HRQOL questionnaires: the EORTC QLQ-C30 and QLQ-HCC18, at their baseline visit before treatment commencement.

\section{EORTC QLQ-C30}

The Chinese version of QLQ-C30 was used [4]. It is a cancer-specific 30-item questionnaire composed of multiple items that reflect the multidimensionality of HRQOL construct, presented in multiple-point Likert scales. These items are grouped into 9 domains and 6 single items. It incorporates 5 functional domains (physical, role, cognitive, emotional and social), 3 symptom domains (fatigue, pain, nausea and vomiting) and a global health and QOL domain. The remaining 6 single items assess additional 5 symptoms commonly reported by cancer patients (dyspnea, appetite loss, sleep disturbance, constipation and diarrhea) as well as perceived financial problem. All domains and scales were converted to scores ranging from 0 to 100 according to the scoring manual [17]. A higher score for a functional or global QOL scale represents a relatively higher/healthier level of functioning or global QOL, whereas a higher score for a symptom/problem scale represents a more severe symptom/problem.

\section{EORTC QLQ-HCC18}

The Chinese version of EORTC QLQ-HCC18 [11] includes 18 multi-item scales. These items are grouped into 6 domains namely fatigue, body image, jaundice, nutrition, pain and fever. Two remaining single items address abdominal swelling and sex life. All scales were grouped and converted to score 0 to 100 according to the scoring manual; a higher score represents a more severe symptom or problem.

\section{$\mathrm{C} 30$ and HCC18 index-scores}

C30 and HCC18 index-scores were derived in order to have an overall representation of all domains/items in QLQ-C30 and QLQ-HCC18 respectively.

To calculate C30 index-score, individual functioning scale was subtracted by 100 to convert them into having the same meaning as symptom/problem scales. These 6 subtracted scores were subsequently summed with the 9 symptom/problem scales, and then divided by 15 (the total number of QLQ-C30 scales). A higher C30 index-score reflects a worse overall HRQOL. This is the mathematical formula:
C30 index-score $=\Sigma[(100$-Physical functioning $)$,

(100-Role functioning), (100-Emotional functioning),

(100-Cognitive functioning), (100-Social functioning),

(100-global QOL), scores of Fatigue, Nausea/vomiting,

Pain, Dyspnoea, Insomnia, Appetite loss, Constipation,

Diarrhea, Financial Diffculty] $\div 15$

HCC18 index-score was defined as the sum of all 8 QLQ-HCC18 symptom/problem scales divided by 8 (the total number of QLQ-HCC18 scales). A higher HCC18 index-score reflects a worse overall HRQOL. This is the mathematical formula:

$\mathrm{HCC} 18$ index - score $=\Sigma($ scores of Fatigue, Body Image, Jaundice, Nutrition, Pain, Fever, Sex life, Abdominal distension) $\div 8$

\section{Clinical factors and follow-up}

Demographic, clinical and laboratory parameters were collected. All patients were followed up for treatment and monitoring until death or last contact.

\section{Statistical analysis}

Standard descriptive analyses were performed to assess sample characteristics. OS was defined as the time from the date-of-consent to date-of-death. In the absence of death confirmation, survival time was censored at the date-of-last-seen. Survival estimation was performed by the Kaplan-Meier method, and compared using the logrank test.

Only patients with complete HRQOL data were included in statistical analysis. EORTC QLQ-C30 and QLQ-HCC18 scales were included in the prognostic factor analysis as (i) continuous variables, (ii) dichotomized $(\geq 50$ or $<50)$ variables, and (iii) index-scores. Univariate analysis was performed with baseline HRQOL scores and non-overlapping clinical variables to identify factors that influenced survival using Cox proportionalhazards regression model. A stepwise model building procedure was used for multivariate analysis, based on a significance value of 0.05 for both inclusion and exclusion of prognostic factors. For analyses involving continuous variables, higher scores (better function and worse symptoms/problems respectively) were compared to lower scores (worse function or better symptoms/problems); whereas for dichotomized data, symptom/problem domain/item scores of $\geq 50$ (worse scores) were compared to $<50$ (better scores), while functional domain scores of $<50$ (worse scores) were compared to $\geq 50$ (better scores).

Treatment options were grouped into curative-intent treatment (surgical/locoablative therapies), palliative-intent treatment (transarterial/systemic therapies) or BSC. 
Performance of the final multivariate models were assessed and compared by Harrell's concordance-index (c-index) [18]. The c-index estimates the probability of concordance between predicted and observed responses. A value of 1.0 indicates perfect separation of patients with different outcomes, and a value of 0.5 indicates no predictive discrimination. Internal validation was carried out by comparing the c-index of each model with the cindexes of 1000 bootstrap replications to obtain optimisms, which were averaged and bootstrap-corrected performance was estimated.

The statistical analyses were performed using SAS version 9.3 software package. A p-value of less than 0.05 was considered significant. The c-index and 95\% confidence intervals $(\mathrm{CI})$ of the different models were calculated by using the SAS macro program.

\section{Results}

\section{Patient characteristics}

Among the 517 patients who consented, 472 (91\%) had complete HRQOL data and were included for analysis. Table 1 listed the clinical characteristics of these patients. The median age at diagnosis was 60 , the majority were male (89\%). Most patients had Eastern Cooperative Oncology Group (ECOG) performance status 0-1 (94\%). $\mathrm{HBV}$ infection was present in $82 \%$, while hepatitis $\mathrm{C}$ in $6 \%$. Fifty nine percent had cirrhosis and $68 \%$ was of ChildPugh class A. Eighteen percent of patients received firstline curative intent treatment, the rest received palliative treatment (44\%) or BSC (38\%).

The median follow-up duration was 29.8 months (95\% CI [26.8-32.8]), 377 patients had died. The median OS was 8.6 months (95\% CI [7.3-10.2]).

Mean scores of QLQ-HCC18 and QLQ-C30 scales and mean $\mathrm{HCC} 18$ and $\mathrm{C} 30$ index-scores were listed in Table 2

\section{Univariate analysis of HRQOL and clinical factors}

Tables 1 and 2 summarized the univariate Cox regression analyses for clinical variables, QLQ-C30 and QLQHCC18 scores, as well as C30 and HCC18 index-scores.

\section{Univariate HRQOL analysis based on continuous variables}

For QLQ-C30, higher (better) scores in all functioning (e.g. physical functioning, HR 0.432 [0.351-0.533]) or global domains were significantly associated with longer OS $(p<0.03)$; whereas higher (worse) scores in fatigue, nausea/vomiting, pain (HR 1.865 [1.584-2.197]), dyspnea, insomnia, appetite loss, diarrhea and financial difficulties were significantly associated with shorter OS $(p<0.01)$.

For QLQ-HCC18, higher (worse) scores in all 8 symptom/problem domains (e.g. fatigue HR 2.381 [1.942-2.919]) were significantly associated with shorter OS $(p<0.05)$.

\section{Univariate QOL analysis based on dichotomization of scores}

For QLQ-C30, scores $\geq 50$ (better) in global QOL, physical, role, cognitive and social functioning and scores $<50$ (better) in all symptom/problem domains (e.g. financial difficulties HR 1.579 [1.276-1.954]) were significantly associated with longer OS $(p<0.05)$.

For QLQ-HCC18, scores $\geq 50$ (worse) in fatigue (HR 2.484 [1.968-3.136]), body image, nutrition, pain, sex life, abdominal swelling domains were significantly associated with shorter OS $(p<0.01)$.

\section{Univariate QOL analysis based on the newly derived index- scores}

Higher C30 index-score (reflecting worse overall functions/symptoms/problems) was significantly associated with shorter OS (HR 3.658 [2.726-4.909] $p<0.0001)$.

Higher HCC18 index-score (reflecting worse overall symptoms/problems) was significantly associated with shorter OS (HR 3.028 [2.340-3.919] $p<0.0001$ ).

\section{Multivariate analysis of HRQOL data with clinical factors} Table 3 shows the results of the multivariate Cox regression analyses involving HRQOL variables or index-scores identified in univariate regression with non-overlapping clinical factors.

\section{Multivariate Analysis of clinical factors}

Multifocal or diffuse HCC, presence of extra-hepatic metastasis, portal vein thrombosis, hypoalbuminemia, hyperbilirubinemia, high AFP, alkaline phosphatase and creatinine were consistently significant clinical factors associated with shorter OS in all multivariate analyses.

Multivariate HRQOL analysis based on continuous variables For QLQ-C30, higher (better) score in physical functioning (HR 0.652 [0.495-0.860], $p=0.0024$ ) was significantly associated with longer OS and higher (worse) score in pain (HR 1.346 [1.092-1.661], $p=0.0055$ ) was significantly associated with shorter OS.

For QLQ-HCC18, higher (worse) scores in fatigue (HR $1.441[1.132-1.833], p=0.0030$ ) and pain (HR 1.382 [1.089-1.754], $p=0.0077)$ were significantly associated with shorter OS.

Multivariate QOL analysis based on dichotomization of scores For QLQ-C30, scores $\geq 50$ (worse) in pain (HR 1.523 [1.192-1.947], $p=0.0008)$, and financial difficulties (HR 1.331 [1.059-1.673], $p=0.0141$ ) and score $<50$ (worse) in physical functioning (HR 1.475 [1.095-1.986], $p=0.0106$ ) were significant independent factors for shorter OS.

For QLQ-HCC18, worse fatigue score ( $\geq 50)$ (HR 1.805 [1.411-2.310], $p<0.0001)$ was significantly associated with shorter OS. 
Table 1 Baseline characteristics and univariate Cox regression analyses of overall survival for patients with complete HRQOL data $(n=472)$

\begin{tabular}{|c|c|c|c|c|c|}
\hline & $n$ & $\%$ & $\mathrm{HR}$ & 95\% C.I. & $p$-value \\
\hline \multicolumn{6}{|l|}{ Demographics/clinical } \\
\hline Age $<=65$ & 328 & 69 & 0.909 & $0.730-1.131$ & 0.3898 \\
\hline Male gender & 419 & 89 & 1.299 & $0.919-1.835$ & 0.1381 \\
\hline$E C O G \geq 2$ & 29 & 6 & 2.885 & $1.927-4.317$ & $<0.0001$ \\
\hline \multicolumn{6}{|l|}{ Laboratory } \\
\hline Hemoglobin $<10 \mathrm{~g} / \mathrm{dL}$ & 27 & 6 & 1.294 & $0.840-1.995$ & 0.2424 \\
\hline White cell count $>10 \times 10^{9} / \mathrm{L}$ & 64 & 14 & 2.507 & $1.885-3.333$ & $<0.0001$ \\
\hline Platelet count $<100 \times 10^{9} / \mathrm{L}$ & 33 & 7 & 1.696 & $1.158-2.485$ & 0.0067 \\
\hline International normalized ratio $>1.4$ & 36 & 8 & 1.410 & $0.945-2.104$ & 0.0922 \\
\hline Creatinine $\geq$ ULN & 67 & 14 & 1.115 & $0.833-1.491$ & 0.4644 \\
\hline Bilirubin $\geq 20 \mathrm{umol} / /$ & 239 & 51 & 1.974 & $1.594-2.445$ & $<0.0001$ \\
\hline Albumin $\leq 35 \mathrm{~g} / \mathrm{l}$ & 182 & 39 & 2.186 & $1.765-2.708$ & $<0.0001$ \\
\hline Alanine aminotransferase $>2 \times U L N$ & 81 & 17 & 1.567 & $1.201-2.044$ & 0.0009 \\
\hline Alkaline phosphatase $>2 \times U L N$ & 145 & 31 & 2.417 & $1.938-3.014$ & $<0.0001$ \\
\hline \multicolumn{6}{|l|}{ Underlying liver condition } \\
\hline Hepatitis B surface antigen + & 386 & 82 & 1.196 & $0.908-1.575$ & 0.2029 \\
\hline Hepatitis C antibody + & 30 & 6 & 0.641 & $0.403-1.019$ & 0.0600 \\
\hline Ascites & 278 & 59 & 0.894 & $0.721-1.107$ & 0.3036 \\
\hline Cirrhosis (radiological) & 122 & 26 & 2.237 & $1.775-2.820$ & $<0.0001$ \\
\hline \multicolumn{6}{|l|}{ Child-Pugh class } \\
\hline A & 319 & 68 & 1.000 & - & - \\
\hline B & 130 & 28 & 2.187 & $1.740-2.750$ & $<0.0001$ \\
\hline $\mathrm{C}$ & 23 & 4 & 4.360 & $2.774-6.854$ & $<0.0001$ \\
\hline \multicolumn{6}{|l|}{ Tumor characteristics } \\
\hline a-fetoprotein $\geq 200 \mathrm{mg} / \mathrm{ml}$ & 250 & 53 & 2.318 & $1.865-2.882$ & $<0.0001$ \\
\hline \multicolumn{6}{|l|}{ Tumor morphology } \\
\hline Uninodular & 122 & 26 & 1.000 & - & - \\
\hline Multinodular & 143 & 30 & 2.097 & $1.509-2.914$ & $<0.0001$ \\
\hline Diffuse & 207 & 44 & 4.360 & $3.027-3.822$ & $<0.0001$ \\
\hline Extrahepatic metastasis (nodal or distant) & 108 & 23 & 3.003 & $2.360-3.822$ & $<0.0001$ \\
\hline Portal vein thrombosis & 152 & 32 & 3.647 & $2.899-4.587$ & $<0.0001$ \\
\hline \multicolumn{6}{|l|}{$1^{\text {st }}$ line Treatment } \\
\hline Curative & 83 & 18 & 1.000 & - & \\
\hline Palliative or best supportive care alone & 389 & 82 & 5.802 & $3.820-8.810$ & $<0.0001$ \\
\hline Surgical treatment & 54 & 12 & 1.000 & - & \\
\hline Local ablative therapies & 29 & 6 & 2.526 & $1.131-5.643$ & 0.0238 \\
\hline Trans-arterial therapies & 116 & 25 & 4.597 & $2.446-8.637$ & $<0.0001$ \\
\hline Systemic therapies & 91 & 19 & 10.209 & $5.380-19.372$ & $<0.0001$ \\
\hline Best supportive care alone & 182 & 38 & 15.624 & $8.442-28.914$ & $<0.0001$ \\
\hline
\end{tabular}

ECOG Eastern Cooperative Oncology Group performance status, ULN upper limit of normal, Cl confidence interval, HR hazard ratio

Figure 1 shows the survival of patients with different scores in significant dichotomized HRQOL domains/ items. The median OS for patients with better $(\geq 50)$ and worse $(<50)$ scores in QLQ-C30 physical functioning were 10.1 (95\%CI $8.6-12.8)$ and 2.3 months (95\% CI $1.9-4.9)$ respectively $(p<0.0001)$. The median OS for patients with better $(<50)$ and worse $(\geq 50)$ scores in QLQ-C30 pain were 13.4 (95\% CI 9.6-16.4) and 3.3 
Table 2 Baseline HRQOL and univariate analyses using continuous, dichotomized variables, C30 and HCC18 index scores for patients with complete HRQOL data $(n=472)^{a}$

\begin{tabular}{|c|c|c|c|c|c|c|}
\hline & Mean & $\pm \mathrm{SD}$ & Continuous variables & & Dichotomized variab & \\
\hline & & & $\mathrm{HR}[95 \% \mathrm{Cl}]$ & $p$-value & $\mathrm{HR}[95 \% \mathrm{Cl}]$ & $p$-value \\
\hline EORTC QLQ-C30 & & & & & & \\
\hline Physical Functioning & 72.27 & 23.74 & $0.432[0.351-0.533]$ & $<0.0001$ & $2.026[1.571-2.612]$ & $<0.0001$ \\
\hline Role Functioning & 74.61 & 32.60 & $0.517[0.443-0.603]$ & $<0.0001$ & $2.108[1.645-2.702]$ & $<0.0001$ \\
\hline Emotional Functioning & 70.67 & 25.48 & $0.777[0.632-0.954]$ & 0.0164 & 1.448 [1.100-1.908] & 0.0084 \\
\hline Social Functioning & 76.80 & 24.68 & $0.698[0.571-0.853]$ & 0.0004 & 1.611 [1.162-2.234] & 0.0042 \\
\hline Cognitive Function & 68.46 & 30.33 & $0.634[0.531-0.756]$ & $<0.0001$ & 1.573 [1.233-2.007] & 0.0003 \\
\hline Global Quality of Life & 52.22 & 26.34 & $0.515[0.417-0.635]$ & $<0.0001$ & 1.611 [1.299-1.998] & $<0.0001$ \\
\hline Fatigue & 42.93 & 30.23 & 1.973 [1.657-2.349] & $<0.0001$ & 2.072 [1.672-2.568] & $<0.0001$ \\
\hline Nausea/Nomiting & 11.26 & 21.41 & $2.050[1.643-2.559]$ & $<0.0001$ & 2.308 [1.679-3.173] & $<0.0001$ \\
\hline Pain & 32.87 & 31.97 & 1.865 [1.584-2.197] & $<0.0001$ & 2.108 [1.698-2.617] & $<0.0001$ \\
\hline Dyspnoea & 29.73 & 31.46 & 1.396 [1.189-1.639] & $<0.0001$ & 1.666 [1.314-2.113] & $<0.0001$ \\
\hline Insomnia & 41.88 & 36.41 & $1.344[1.162-1.556]$ & $<0.0001$ & 1.415 [1.144-1.750] & 0.0014 \\
\hline Appetite loss & 32.34 & 35.88 & $1.923[1.668-2.217]$ & $<0.0001$ & 2.360 [1.889-2.949] & $<0.0001$ \\
\hline Constipation & 16.67 & 27.13 & 1.201 [0.999-1.444] & 0.0512 & 1.368 [1.021-1.834] & 0.0359 \\
\hline Diarrhea & 16.45 & 26.87 & $1.520[1.252-1.845]$ & $<0.0001$ & 1.666 [1.248-2.224] & 0.0005 \\
\hline Financial difficulties & 51.20 & 37.22 & 1.353 [1.169-1.566] & $<0.0001$ & 1.579 [1.276-1.954] & $<0.0001$ \\
\hline C30 index score & 30.69 & 19.61 & 3.658 [2.726-4.909] & $<0.0001$ & - & - \\
\hline EORTC QLQ-HCC18 & & & & & & \\
\hline Fatigue & 35.23 & 25.86 & 2.381 [1.942-2.919] & $<0.0001$ & 2.484 [1.968-3.136] & $<0.0001$ \\
\hline Body Image & 25.35 & 22.98 & 2.261 [1.819-2.811] & $<0.0001$ & 2.167 [1.718-2.733] & $<0.0001$ \\
\hline Jaundice & 23.41 & 22.15 & 1.289 [1.024-1.623] & 0.0307 & $1.271[0.977-1.652]$ & 0.0739 \\
\hline Nutrition & 26.96 & 21.35 & 2.934 [2.317-3.716] & $<0.0001$ & 2.663 [2.026-3.502] & $<0.0001$ \\
\hline Pain & 23.34 & 24.57 & 2.107 [1.717-2.587] & $<0.0001$ & $1.871[1.465-2.391]$ & $<0.0001$ \\
\hline Fever & 6.60 & 14.39 & 1.568 [1.123-2.187] & 0.0081 & 1.697 [0.929-3.099] & 0.0851 \\
\hline Sex life & 28.74 & 34.76 & $1.213[1.040-1.415]$ & 0.0139 & 1.436 [1.128-1.828] & 0.0033 \\
\hline Abdominal swelling & 33.33 & 35.43 & 1.721 [1.486-1.994] & $<0.0001$ & 2.192 [1.752-2.743] & $<0.0001$ \\
\hline HCC18 index score & 25.37 & 17.21 & 3.028 [2.340-3.919] & $<0.0001$ & - & - \\
\hline
\end{tabular}

C30 index-score $=\Sigma$ [(100-Physical functioning), (100-Role functioning), (100-Emotional functioning), (100-Cognitive functioning), (100-Social functioning), (100-global $\mathrm{QOL}$ ), scores of Fatigue, Nausea/vomiting, Pain, Dyspnoea, Insomnia, Appetite loss, Constipation, Diarrhea, Financial Diffculty] $\div 15$

$\mathrm{HCC1} 18$ index-score $=\Sigma$ (scores of Fatigue, Body Image, Jaundice, Nutrition, Pain, Fever, Sex life, Abdominal distension) $\div 8$

${ }^{a}$ In dichotomization, worse ( $\geq 50$ in symptoms/problem or $<50$ in functioning/global QOL) scores in QLQ-C30 were analyzed with respect to better scores; worse $(\geq 50)$ scores in QLQ-HCC18 were analyzed with respect to better scores

$S D$ standard deviation, $C l$ confidence interval, HR hazard ratio, HRQOL health-related quality of life

months (95\%CI 2.7-4.7) respectively $(p<0.0001)$, that of QLQ-C30 financial difficulties were $12.8(95 \% \mathrm{CI}$ 9.8-15.9) and 5.2 months (95\% CI 4.0-7.8) $(p<0.0001)$ and QLQ-HCC18 fatigue were 12.1 (95\% CI 9.8-14.8) and 2.6 months $(95 \%$ CI 1.9-3.1) $(p<0.0001)$ respectively.

\section{Multivariate QOL analysis based on the newly derived index-scores}

In the multivariate analysis using C30 index-score with clinical factors, higher (worse) C30 index-score was a significant independent risk factor for shorter OS (HR 2.143 [1.616-2.841], $p<0.0001)$.
In the multivariate analysis using HCC18 index-score with clinical factors, higher (worse) HCC18 index-score was a significant independent risk factor for shorter OS (HR 1.957 [1.411-2.715], $p<0.0001$ ).

Figure 2 shows the OS plots for patients with stratified C30 and HCC18 index-scores respectively. Lower (better) C30 index-score ranges were associated with longer OS in a step-wise fashion $(p<0.0001)$; similarly, the lower (better) HCC18 index-score ranges, the longer the OS $(p<0.0001)$.

The median OS in patients with C30 index-score of 0-20 was 16.4 (95\% CI 13.4-22.3) months, that for score 21-40 was 7.3 (95\% CI 4.5-10.4) months, score 41-60 
Table 3 Multivariate analysis of HRQOL variables or index scores with significant clinical factors $(n=472)$

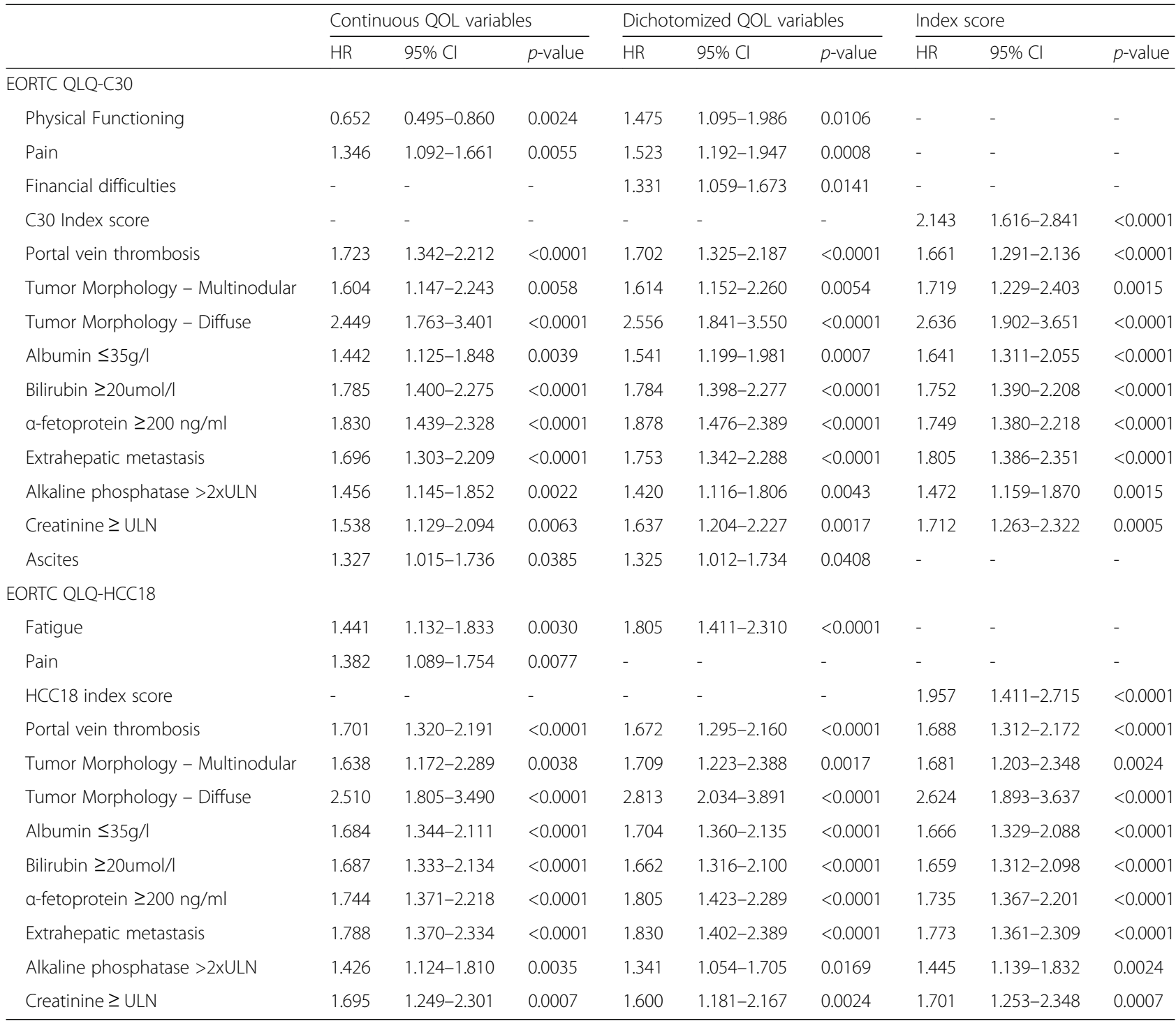

ULN upper limit of normal, $\mathrm{Cl}$ confidence interval, $H R$ hazard ratio, $H R Q O L$ health-related quality of life

was 3.1 (95\%CI 2.3-5.1) months, score 61-80 was 1.8 (95\% CI $0.8-4.3)$ months, and score $81-100$ was 1.8 (95\% CI 0.5-6) months $(p<0.0001)$. The median OS of patients with HCC18 index-score of $0-20$ was 16.4 (95\% CI 31-not reached) months, that for score 21-40 was 5.9 (95\%CI 4.4-8.9) months, score 41-60 was 2.9 (95\% CI 1.6-4.3) months, score $61-80$ was 1.8 (95\% CI $0.5-3.0)$ months, and score $81-100$ was 1.8 (95\% CI $0.7-2.9$ ) months $(p<0.0001)$ (Table 4$)$.

\section{Internal validation of the multivariate cox proportional hazard models}

C-index of original dataset and mean c-index of 1000 bootstrap samples for multivariate model are described below. Using QLQ-C30 as continuous variables, the corresponding values were 0.7872 (95\% CI $0.7648-0.8905$ ) and 0.7891 (95\% CI $0.7678-0.8111$ ) respectively. Using QLQ-C30 as dichotomized variables, the values were 0.7842 (95\% CI $0.7617-0.8066)$ and 0.7878 (95\% CI $0.7660-0.8103)$ respectively. When assessed using C30 index score, the values were 0.7817 (95\%CI $0.7591-$ $0.8043)$ and 0.7840 (95\% CI $0.7626-0.8066)$ respectively. Using QLQ-HCC18 as continuous variables, the values were 0.7810 (95\% CI $0.7588-0.8032)$ and 0.7841 (95\% CI 0.7638-0.8056) respectively. For QLQ-HCC18 as dichotomized variables, the values were 0.7821 (95\%CI $0.7598-0.8043$ ) and 0.7839 (95\% CI $0.7621-$ 0.8072 ) respectively. For HCC18 index score, the values were 0.7791 (95\% CI 0.7564-0.8018) and 0.7715 (95\% CI 0.7604-0.8034) respectively. All optimisms were within \pm 0.01 (Table 5). The internally validated optimismcorrected c-index was estimated to be 0.78 . 


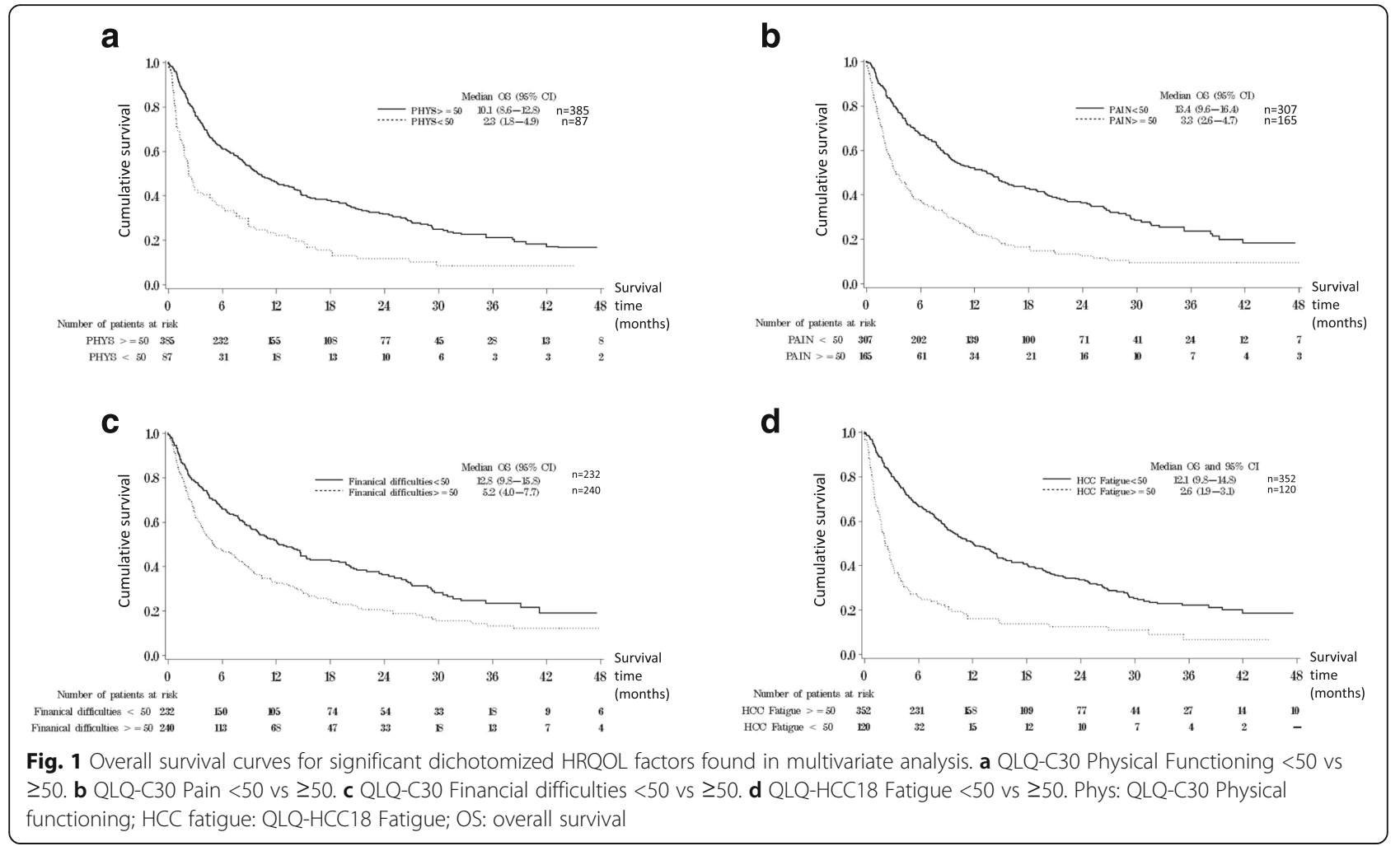

Multiple comparisons showed no statistically significant difference in c-index among related multivariate cox proportional hazard models (Table 6).

\section{Discussion}

This is the first prospective study to demonstrate that the prognostic significance of QLQ-C30 was not limited to advance-stage HCC patients but valid for newly diagnosed patients with various stages of disease. Worse scores in physical functioning, pain and financial difficulties were associated with shorter OS in dichotomized variable analyses, while worse scores in physical functioning and pain were significant in continuous variable analyses.
This is also the first prospective study to demonstrate that baseline QLQ-HCC18 is a significant prognostication tool for OS in newly diagnosed HCC patients. Worse dichotomized score in fatigue was an independent prognostic factor for shorter OS, while worse continuous scores in fatigue and pain were also significant poor prognostic factors.

Physical functioning domain in the present study concurred with previous findings by Yeo et al. [1] (where physical and role functioning, appetite loss were significant prognostic factors for OS), and Diouf et al. [3] (where physical [dichotomized] or role functioning [continuous] were significant factors).

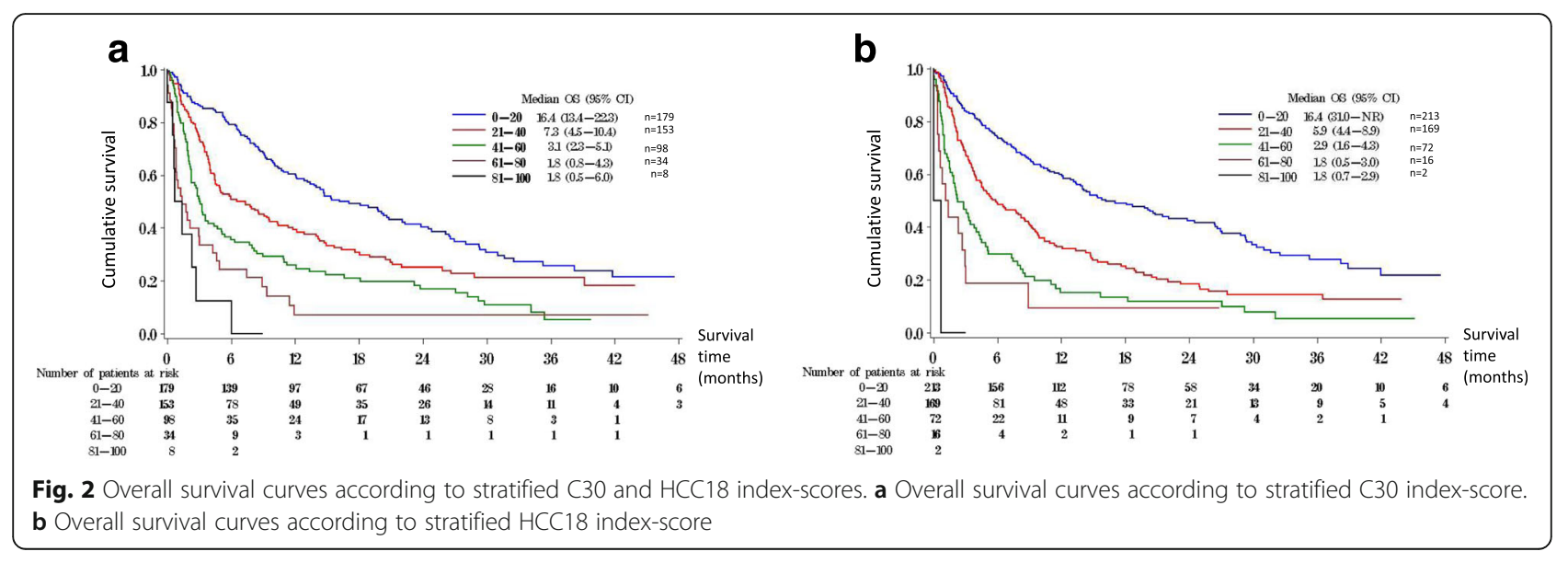


Table 4 Overall survival data for patients stratified according to C30 and HCC18 index score range $(n=472)$

\begin{tabular}{|c|c|c|c|c|c|c|c|}
\hline & $N$ & Median OS (M) & $95 \% \mathrm{Cl}$ & Survival\% at $6 \mathrm{M}$ & Survival\% at $12 \mathrm{M}$ & Survival\% at $24 \mathrm{M}$ & Survival\% at $36 \mathrm{M}$ \\
\hline \multicolumn{8}{|c|}{ C30 Index Score } \\
\hline $0-20$ & 179 & 16.4 & $13.4-22.3$ & 79.7 & 60.4 & 41.3 & 25.6 \\
\hline $21-40$ & 153 & 7.3 & $4.5-10.4$ & 52.0 & 39.2 & 26.0 & 21.4 \\
\hline $41-60$ & 98 & 3.1 & $2.3-5.1$ & 36.5 & 27.0 & 18.3 & 8.1 \\
\hline $61-80$ & 34 & 1.8 & $0.8-4.3$ & 27.5 & 10.7 & 7.1 & 7.1 \\
\hline $81-100$ & 8 & 1.8 & $0.5-6.0$ & 25.0 & - & - & - \\
\hline \multicolumn{8}{|c|}{ HCC18 Index Score } \\
\hline $0-20$ & 213 & 16.4 & 31.0-NR & 74.6 & 59.9 & 43.1 & 27.8 \\
\hline $21-40$ & 169 & 6.0 & $4.4-8.9$ & 49.6 & 33.1 & 19.3 & 14.5 \\
\hline $41-60$ & 72 & 2.8 & $1.6-4.3$ & 31.1 & 16.7 & 11.8 & 5.3 \\
\hline $61-80$ & 16 & 1.8 & $0.5-3.0$ & 25.0 & 18.8 & 9.4 & - \\
\hline $81-100$ & 2 & 1.8 & $0.7-2.9$ & - & - & - & - \\
\hline
\end{tabular}

$M$ month (s), $C l$, confidence interval, $N R$ not reached

The HRQOL factors identified in this study varied from previous studies and could be due to a number of reasons. Firstly, patient populations were different, our study involved early as well as advanced stage HCC patients while prior studies involved only advanced stage disease. Secondly, patients of different cultural backgrounds could have different HRQOL perceptions. Thirdly, studies conducted more recently carried more available treatment options than earlier era, which may have led to differences in perception of disease and thus HRQOL. Fourthly, although different studies might utilize the same HRQOL tool, the methodologies of data analysis varied across trials.

The failure to identify consistent HRQOL factors for OS across studies makes clinically meaningful utilization of HRQOL for prognostication difficult. On the other hand, using simple algorithm and calculation, C30 and HCC18 index-scores could be derived from the raw data of all domains and items within QLQ-C30 and QLQ-
HCC18 respectively. It is a meaningful representation of the overall HRQOL of an individual patient.

The C30 and HCC18 index-scores were proven to be highly significant prognostic factors for survival, and were more significant than any individual HRQOL factor, whether continuous or dichotomized. When the index-scores were stratified into subgroups, distinct OS outcomes could be identified. Clinical use of either C30 or HCC18 index-score at baseline provides another means of survival estimation in patients with newly diagnosed HCC apart from conventional staging systems. Index-score could be calculated in the clinical setting in a user-friendly manner. With the aid of modern computer technology, patients may be able to self-administer the QLQ-C30 or QLQ-HCC18 questionnaire and have the respective index-score generated by handheld devices.

One limitation of this study was the lack of a separate patient population, for instance, that of a different geographical or cultural background, to allow external

Table 5 Performance and internal validation of all the multivariate cox proportional hazard models

\begin{tabular}{lllllll}
\hline MV model & c-index & $95 \% \mathrm{Cl}$ & $\begin{array}{l}\text { Mean c-index from } \\
\text { 1000 bootstraps }\end{array}$ & $\begin{array}{l}95 \% \mathrm{Cl} \text { based on } 1000 \\
\text { bootstrap samples }\end{array}$ & Optimism & Optimism in \% \\
\hline M1 & 0.7872 & $0.7648-0.8095$ & 0.7891 & $0.7678-0.8111$ & 0.0019 & $0.24 \%$ \\
M2 & 0.7842 & $0.7617-0.8066$ & 0.7878 & $0.7660-0.8103$ & 0.0036 & $0.46 \%$ \\
M3 & 0.7817 & $0.7591-0.8043$ & 0.7840 & $0.7626-0.8066$ & 0.0023 & $0.29 \%$ \\
M4 & 0.7810 & $0.7588-0.8032$ & 0.7841 & $0.7638-0.8056$ & 0.0031 & $0.40 \%$ \\
M5 & 0.7821 & $0.7598-0.8043$ & 0.7839 & $0.7621-0.8072$ & 0.0018 & $0.23 \%$ \\
M6 & 0.7791 & $0.7564-0.8018$ & 0.7715 & $0.7604-0.8034$ & -0.0076 & $-0.96 \%$ \\
\hline
\end{tabular}

MV multivariate, $\mathrm{Cl}$ confidence interval

M1: the multivariate cox model using QLQ-C30 as continuous variables

M2: the multivariate cox model using QLQ-C30 as dichotomized variables

M3: the multivariate cox model using C30 index score

M4: the multivariate cox model using QLQ-HCC18 as continuous variables

M5: the multivariate cox model using QLQ-HCC18 as dichotomized variables

M6: the multivariate cox model using HCC18 index score 
Table 6 Multiple comparison of c index among various multivariate cox proportional hazard models

\begin{tabular}{lll}
\hline MV model & MV model & p-value \\
\hline M1 & M2 & 0.93067 \\
M1 & M3 & 0.74456 \\
M2 & M3 & 0.81230 \\
M4 & M5 & 0.99435 \\
M4 & M6 & 0.86892 \\
M5 & M6 & 0.87639 \\
M1 & M4 & 0.74687 \\
M2 & M5 & 0.81263 \\
M3 & M6 & 0.87526 \\
\hline
\end{tabular}

MV multivariate

M1: the multivariate cox model using QLQ-C30 as continuous variables M2: the multivariate cox model using QLQ-C30 as dichotomized variables M3: the multivariate cox model using C30 index score

M4: the multivariate cox model using QLQ-HCC18 as continuous variables M5: the multivariate cox model using QLQ-HCC18 as dichotomized variables M6: the multivariate cox model using HCC18 index score

validation of the multivariate cox proportional hazard models. However, bootstrapping has enabled internal validation of the multivariate models.

HRQOL assessment is important to aid clinical management in $\mathrm{HCC}$ patients. Being an aggressive disease, patients commonly present at advanced stage when treatment option is limited, of modest benefit and associated with disabling toxicities. HRQOL assessment enables the identification of symptoms/problems, whereby symptom control and psychosocial support measures could be offered as part of palliative care in parallel with anti-neoplastic therapy.

HRQOL tools could further be utilized to provide prognostic information. HRQOL analyses may potentially supplement available clinical staging systems in prognostication. External validation of the role of QLQC30 and HCC18 index-scores in prognostication in HCC patients is warranted. Index-scoring may prove useful in HRQOL research for other cancer types and further studies are encouraged.

\section{Conclusions}

Both EORTC QLQ-HCC18 and QLQ-C30 measurements at presentation are prognostic for OS in newly diagnosed patients with $\mathrm{HCC}$ of various stages. Index-scores of QLQHCC18 and QLQ-C30 are highly significant prognostic factors for OS in newly diagnosed HCC patients. Indexscoring provides an effective way to summarize, analyze and interpret raw HRQOL data, and renders QLQ-HCC18 and QLQ-C30 meaningful and communicable in clinical practice. Index-scores of both EORTC QLQ-C30 and QLQ-HCC18 could potentially serve as a standardized tool for future HRQOL research.

\section{Abbreviations}

AFP: a-fetoprotein; BSC: Best supportive care; ECOG: Eastern Cooperative Oncology Group; EORTC: European Organization for Research and Treatment of Cancer; FACT-G: Functional Assessment of Cancer Therapy - General; HBV: Hepatitis B virus; HCC: Hepatocellular carcinoma; HRQOL: Health-related quality-of-life; LEM: Transarterial injection of lipiodol-ethanol mixture; OS: Overall survival; PEl: Percutaneous ethanol injection; RFA: Radiofrequency ablation; TACE: Transarterial chemo-embolisation

\section{Acknowledgement}

Not applicable.

Funding

Not applicable.

\section{Availability of data and materials}

Dataset of the study contains individual privacy and is therefore not publicly available. The dataset upon request could be provided at the discretion of the investigators.

\section{Authors' contributions}

$W Y^{1}, \mathrm{LL}^{1}$ designed research directions. $W Y, L L, J K^{1}, S L C^{1}, E P H^{1}, B B Y M^{1}, \mathrm{LKSL}^{1}$, ANYP ${ }^{1}, \mathrm{CMC}^{2}, \mathrm{JH}^{2}, \mathrm{KFL}^{3}, \mathrm{PBSL}^{3}, \mathrm{NSLT}^{4}, \mathrm{ATCC}^{1}$ and $\mathrm{SCHY}^{2}$ acquired clinical, radiology and laboratory data. $\mathrm{WY}, \mathrm{LL}, \mathrm{FKFM}^{1}$ analyzed and interpreted data. WY, LL wrote the manuscript. All authors read, revised and approved the final manuscript.

\section{Competing interests}

The authors declare that they have no competing interests.

\section{Consent for publication}

There is no individual person's data included in the publication.

\section{Ethics approval and consent to participate}

The study was approved by the Joint Chinese University of Hong Kong-New Territories East Cluster Clinical Research Ethics Committee. All participants consented to participate in the study and signed a written informed consent.

\section{Author details}

'Comprehensive Cancer Trials Unit, Department of Clinical Oncology, State Key Laboratory in Oncology in South China, Prince of Wales Hospital, Faculty of Medicine, The Chinese University of Hong Kong, Shatin, Hong Kong SAR. 2Department of Diagnostic and Interventional Radiology, Prince of Wales Hospital, Shatin, Hong Kong SAR. ${ }^{3}$ Department of Surgery, Prince of Wales Hospital, Shatin, Hong Kong SAR. ${ }^{4}$ Department of Chemical Pathology, Li Ka Shing Institute of Health Sciences, Faculty of Medicine, The Chinese

University of Hong Kong, Shatin, Hong Kong SAR.

Received: 8 January 2016 Accepted: 13 December 2016

Published online: 04 January 2017

References

1. Yeo W, Mo FK, Koh J, Chan AT, Leung T, Hui P, Chan L, Tang A, Lee JJ, Mok TS, et al. Quality of life is predictive of survival in patients with unresectable hepatocellular carcinoma. Ann Oncol. 2006;17(7):1083-9.

2. Bonnetain F, Paoletti X, Collette S, Doffoel M, Bouche $O$, Raoul JL, Rougier $P$, Masskouri F, Barbare JC, Bedenne L. Quality of life as a prognostic factor of overall survival in patients with advanced hepatocellular carcinoma: results from two French clinical trials. Qual Life Res. 2008;17(6):831-43.

3. Diouf M, Filleron T, Barbare JC, Fin L, Picard C, Bouche O, Dahan L, Paoletti $X$, Bonnetain $F$. The added value of quality of life (QoL) for prognosis of overall survival in patients with palliative hepatocellular carcinoma. J Hepatol. 2013;58(3):509-21.

4. Aaronson NK, Ahmedzai S, Bergman B, Bullinger M, Cull A, Duez NJ, Filiberti A, Flechtner H, Fleishman SB, de Haes JC, et al. The European Organization for Research and Treatment of Cancer QLQ-C30: a quality-of-life instrument for use in international clinical trials in oncology. J Natl Cancer Inst. 1993;85(5):365-76. 
5. Spitzer WO, Dobson AJ, Hall J, Chesterman E, Levi J, Shepherd R, Battista RN, Catchlove BR. Measuring the quality of life of cancer patients: a concise QLindex for use by physicians. J Chronic Dis. 1981;34(12):585-97.

6. Fielding $R$, Wong WS. Quality of life as a predictor of cancer survival among Chinese liver and lung cancer patients. Eur J Cancer. 2007;43(11):1723-30.

7. Cella DF, Tulsky DS, Gray G, Sarafian B, Linn E, Bonomi A, Silberman M, Yellen SB, Winicour P, Brannon J, et al. The Functional Assessment of Cancer Therapy scale: development and validation of the general measure. J Clin Oncol. 1993;11(3):570-9.

8. Chin PL, Chu DZ, Clarke KG, Odom-Maryon T, Yen Y, Wagman LD. Ethnic differences in the behavior of hepatocellular carcinoma. Cancer. 1999;85(9):1931-6.

9. Lok AS, Lai CL, Wu PC, Wong VC, Yeoh EK, Lin HJ. Hepatitis B virus infection in Chinese families in Hong Kong. Am J Epidemiol. 1987;126(3):492-9.

10. Ho J, Wu PC, Kung TM. An autopsy study of hepatocellular carcinoma in Hong Kong. Pathology. 1981;13(3):409-16.

11. Blazeby JM, Currie E, Zee BC, Chie WC, Poon RT, Garden OJ. Development of a questionnaire module to supplement the EORTC QLQ-C30 to assess quality of life in patients with hepatocellular carcinoma, the EORTC QLQHCC18. Eur J Cancer. 2004;40(16):2439-44.

12. Chie WC, Blazeby JM, Hsiao CF, Chiu HC, Poon RT, Mikoshiba N, Al-Kadhimi G, Heaton N, Calara J, Collins P, et al. International cross-cultural field validation of an European Organization for Research and Treatment of Cancer questionnaire module for patients with primary liver cancer, the European Organization for Research and Treatment of Cancer quality-of-life questionnaire HCC18. Hepatology. 2012:55(4):1122-9.

13. Mikoshiba N, Tateishi R, Tanaka M, Sakai T, Blazeby JM, Kokudo N, Koike K, Kazuma K. Validation of the Japanese version of the EORTC hepatocellular carcinoma-specific quality of life questionnaire module (QLQ-HCC18). Health Qual Life Outcomes. 2012;10:58.

14. Gotay CC, Kawamoto CT, Bottomley A, Efficace F. The prognostic significance of patient-reported outcomes in cancer clinical trials. J Clin Oncol. 2008:26:1355-63.

15. Van Steen K, Curran D, Kramer J, Molenberghs G, Van Vreckem A, Bottomley A, Sylvester R. Multicollinearity in prognostic factor analyses using the EORTC QLQ-C30: identification and impact on model selection. Stat Med. 2002;21(24):3865-84

16. Diouf M, Bonnetain F, Barbare JC, Bouche O, Dahan L, Paoletti X, Filleron T. Optimal cut points for quality of life questionnaire-core 30 (QLQ-C30) scales: utility for clinical trials and updates of prognostic systems in advanced hepatocellular carcinoma. Oncologist. 2015;20(1):62-71.

17. Fayers P. EORTC QLQ-C30 scoring manual. 2nd ed. EORTC: EORTC Data Center; 1999.

18. Harrell Jr FE, Lee KL, Mark DB. Multivariable prognostic models: issues in developing models, evaluating assumptions and adequacy, and measuring and reducing errors. Stat Med. 1996;15(4):361-87.

\section{Submit your next manuscript to BioMed Central and we will help you at every step:}

- We accept pre-submission inquiries

- Our selector tool helps you to find the most relevant journal

- We provide round the clock customer support

- Convenient online submission

- Thorough peer review

- Inclusion in PubMed and all major indexing services

- Maximum visibility for your research

Submit your manuscript at www.biomedcentral.com/submit
Biomed Central 